\title{
Recent Progress in the Theory of Nuclear Forces
}

\author{
R. Machleidt • Q. MacPherson • E. Marji • \\ R. Winzer • Ch. Zeoli • D. R. Entem
}

Received: date / Accepted: date

\begin{abstract}
During the past two decades, it has been demonstrated that chiral effective field theory represents a powerful tool to deal with nuclear forces in a systematic and model-independent way. Two-, three-, and four-nucleon forces have been derived up to next-to-next-to-next-to-leading order $\left(\mathrm{N}^{3} \mathrm{LO}\right)$ and (partially) applied in nuclear few- and many-body systems - with, in general, a good deal of success. This may suggest that we are finally done with the nuclear force problem; but that would be too optimistic. There are still some pretty basic open issues that have been swept under rug and, finally, need our full attention, like the proper renormalization of the twonucleon potential. Moreover, the order-by-order convergence of the many-body force contributions is at best obscure at this time.
\end{abstract}

Keywords Effective field theory · Chiral perturbation theory · Renormalization · Nuclear two- and many-body forces

\section{Introduction}

The theory of nuclear forces has a long history (cf. Table 1). Based upon the seminal idea by Yukawa [1, first field-theoretic attempts to derive the nucleon-nucleon $(N N)$ interaction focused on pion exchange. While the one-pion exchange turned out to be very useful in explaining $N N$ scattering data and, in particular, the properties of the

Presented at the 20th International IUPAP Conference on Few-Body Problems in Physics, 20 - 25 August, 2012, Fukuoka, Japan

R. Machleidt · Q. MacPherson · E. Marji · R. Winzer · Ch. Zeoli

Department of Physics, University of Idaho, Moscow, ID 83844-0903, USA

E-mail: machleid@uidaho.edu

Present address: Ch. Zeoli

Department of Physics, Florida State University, Tallahassee, FL 32306-4350, USA

Present address: E. Marji

College of Western Idaho, Nampa, ID 83653, USA

D. R. Entem

Grupo de Fisica Nuclear and IUFFyM, University of Salamanca, E-37008 Salamanca, Spain E-mail: entem@usal.es 
Table 1 The Theory of Nuclear Forces: Eight Decades of Struggle

\begin{tabular}{|c|c|}
\hline 1935 & Yukawa: Meson Theory \\
\hline 1950 's & $\begin{array}{l}\text { The "Pion Theories". } \\
\text { One-pion exchange: good; } \\
\text { Multi-pion exchange: disaster. }\end{array}$ \\
\hline 1960's & $\begin{array}{l}\text { Many pions } \equiv \text { multi-pion resonances: } \boldsymbol{\sigma}, \boldsymbol{\rho}, \boldsymbol{\omega}, \ldots \\
\text { The One-Boson-Exchange Model: success. }\end{array}$ \\
\hline 1970's & $\begin{array}{c}\text { Refinement of meson theory: Sophisticated } 2 \pi \text { exchange models; } \\
\text { Partovi-Lomon, Stony Brook, Paris, Bonn. }\end{array}$ \\
\hline 1980's & $\begin{array}{l}\text { Nuclear physicists discover QCD: } \\
\text { Quark Models. }\end{array}$ \\
\hline $\begin{array}{l}1990 \text { 's } \\
\text { and beyond }\end{array}$ & $\begin{array}{c}\text { Nuclear physicists discover EFT; Weinberg, van Kolck, ... } \\
\text { Back to Yukawa's Meson (Pion) Theory! } \\
\text { But, constrained by Chiral Symmetry. }\end{array}$ \\
\hline
\end{tabular}

deuteron [2], multi-pion exchange was beset with serious ambiguities 3 that could not be resolved in a satisfactory way. Thus, the "pion theories" of the 1950s are generally judged as failures - for reasons we understand today: pion dynamics is constrained by chiral symmetry, a crucial point that was unknown in the 1950s.

Historically, the experimental discovery of heavy mesons in the early 1960 s saved the situation. The one-boson-exchange (OBE) model emerged, which still today is the most economical and quantitative phenomenology for describing the $N N$ interaction [4]. The weak point of this model, however, is the scalar-isoscalar "sigma" or "epsilon" boson, for which empirical evidence remains controversial. Since this boson is associated with the correlated (or resonant) exchange of two pions, a vast theoretical effort was launched to derive the $2 \pi$-exchange contribution of the nuclear force, which creates the intermediate range attraction. During this effort, which occupied more than a decade, dispersion theory (Stony Brook and Paris potentials) as well as field theory (PartoviLomon model, Bonn potential [4,5]) were invoked.

The nuclear force problem appeared to be solved; however, with the discovery of quantum chromo-dynamics (QCD), all "meson theories" were relegated to the status of models and the attempts to derive the nuclear force had to start all over again.

The problem with a derivation of nuclear forces from QCD is that this theory is non-perturbative in the low-energy regime characteristic of nuclear physics, which makes direct solutions very difficult. Therefore, during the first round of new attempts, QCD-inspired quark models became popular. The positive aspect of these models is that they try to explain hadron structure and hadron-hadron interactions on an equal footing and, indeed, some of the gross features of the $N N$ interaction are explained successfully. However, on a critical note, it must be pointed out that these quarkbased approaches are nothing but another set of models and, thus, do not represent fundamental progress. For the purpose of describing hadron-hadron interactions, one may equally well stay with the simpler and much more quantitative meson models.

A major breakthrough occurred when the concept of an effective field theory (EFT) was introduced and applied to low-energy QCD. As outlined by Weinberg in a seminal paper [6], one has to write down the most general Lagrangian consistent with the 
assumed symmetry principles, particularly the (broken) chiral symmetry of QCD. At low energy, the effective degrees of freedom are pions (the Goldstone bosons of the broken symmetry) and nucleons rather than quarks and gluons; heavy mesons and nucleon resonances are "integrated out". So, the circle of history is closing and we are back to Yukawa's meson (pion) theory, except that we have finally learned how to deal with it: broken chiral symmetry is a crucial constraint that generates and controls the dynamics and establishes a clear connection with the underlying theory, QCD.

The past 15 years have seen great progress in applying chiral perturbation theory (ChPT) to nuclear forces $[7,8,9,10,11,12,13,14$. As a result, nucleon-nucleon $(N N)$ potentials of high precision have been constructed, which are based upon ChPT carried to next-to-next-to-next-to-leading order $\left(\mathrm{N}^{3} \mathrm{LO}\right)$ [12,13,14], and applied in nuclear structure calculations with great success.

However, in spite of this progress, we are not done. Due to the complexity of the nuclear force issue, there are still many subtle and not so subtle open problems. We will not list and discuss all of them, but instead just focus on the two open issues, which we perceive as the most important ones:

- The proper renormalization of chiral nuclear potentials and

- Subleading chiral few-nucleon forces.

\section{Renormalization of chiral nuclear forces}

2.1 The chiral $N N$ potential

In terms of naive dimensional analysis or "Weinberg counting", the various orders of the irreducible graphs which define the chiral $N N$ potential are given by:

$$
\begin{aligned}
V_{\mathrm{LO}} & =V_{\mathrm{ct}}^{(0)}+V_{1 \pi}^{(0)} \\
V_{\mathrm{NLO}} & =V_{\mathrm{LO}}+V_{\mathrm{ct}}^{(2)}+V_{1 \pi}^{(2)}+V_{2 \pi}^{(2)} \\
V_{\mathrm{NNLO}} & =V_{\mathrm{NLO}}+V_{1 \pi}^{(3)}+V_{2 \pi}^{(3)} \\
V_{\mathrm{N} 3} \mathrm{LO} & =V_{\mathrm{NNLO}}+V_{\mathrm{ct}}^{(4)}+V_{1 \pi}^{(4)}+V_{2 \pi}^{(4)}+V_{3 \pi}^{(4)}
\end{aligned}
$$

where the superscript denotes the order $\nu$ of the low-momentum expansion. LO stands for leading order, NLO for next-to-leading order, etc.. Contact potentials carry the subscript "ct" and pion-exchange potentials can be identified by an obvious subscript. For more details concerning the above potentials, see ref. 14.

\subsection{Nonperturbative renormalization of the $N N$ potential}

The two-nucleon system is characterized by large scattering lengths and shallow (quasi) bound states which require a nonperturbative treatment. Following Weinberg's prescription [7, this is accomplished by inserting the potential $V$ into the LippmannSchwinger (LS) equation:

$$
T\left(\mathbf{p}^{\prime}, \mathbf{p}\right)=V\left(\mathbf{p}^{\prime}, \mathbf{p}\right)+\int d^{3} p^{\prime \prime} V\left(\mathbf{p}^{\prime}, \mathbf{p}^{\prime \prime}\right) \frac{M_{N}}{p^{2}-p^{\prime \prime 2}+i \epsilon} T\left(\mathbf{p}^{\prime \prime}, \mathbf{p}\right),
$$

where $M_{N}$ denotes the nucleon mass. 
In general, the integral in the LS equation is divergent and needs to be regularized. One way to achieve this is by multiplying $V$ with a regulator function

$$
V\left(\mathbf{p}^{\prime}, \mathbf{p}\right) \longmapsto V\left(\mathbf{p}^{\prime}, \mathbf{p}\right) \boldsymbol{e}^{-\left(p^{\prime} / \Lambda\right)^{2 n}} \boldsymbol{e}^{-(p / \Lambda)^{2 n}}
$$

Typical choices for the cutoff parameter $\Lambda$ that appears in the regulator are $\Lambda \approx$ $0.5 \mathrm{GeV}<\Lambda_{\chi} \approx 1 \mathrm{GeV}$.

In field theories, divergent integrals are not uncommon and methods have been designed to deal with them. One regulates the integrals and then removes the dependence on the regularization parameters (scales, cutoffs) by "renormalization". In the end, the theory and its predictions do not depend on cutoffs or renormalization scales. So-called renormalizable quantum field theories, like QED, have essentially one set of prescriptions that takes care of renormalization through all orders. In contrast, EFTs are renormalized by "counter terms" (contact terms) that are introduced order by order in increasing numbers.

Naively, the most perfect renormalization procedure is the one where the cutoff parameter $\Lambda$ is taken to infinity while stable and quantitative results are maintained through the adjustment of counter terms. This was accomplished at LO in the work by Nogga et al [15]. At NNLO, the infinite-cutoff renormalization procedure has been investigated in [16] for partial waves with total angular momentum $J \leq 1$ and in 17] for all partial waves with $J \leq 5$. However, for a quantitative chiral $N N$ potential one needs to advance all the way to $\mathrm{N}^{3} \mathrm{LO}$. At $\mathrm{N}^{3} \mathrm{LO}$, the ${ }^{1} S_{0}$ state was considered in Ref. 18, and all states up to $J=6$ were investigated in Ref. 19. From all of these works, it is evident that no counter term is effective in partial-waves with short-range repulsion and only a single counter term can constructively be used in partial-waves with short-range attraction. Thus, for the $\Lambda \rightarrow \infty$ renormalization prescription, even at $\mathrm{N}^{3} \mathrm{LO}$, there exists either one or no counter term per partial-wave state. This is inconsistent with any reasonable power-counting scheme and prevents an order-by-order improvement of the predictions.

To summarize: In the infinite-cutoff renormalization scheme, the potential is admitted up to unlimited momenta. However, the EFT this potential is derived from has validity only for momenta smaller than the chiral symmetry breaking scale $\Lambda_{\chi} \approx$ $\mathrm{GeV}$. The lack of order-by-order convergence and discrepancies in lower partial-waves demonstrate that the potential should not be used beyond the limits of the effective theory 19 (see Ref. 20 for a related discussion). The conclusion then is that cutoffs should be limited to $\Lambda \lesssim \Lambda_{\chi}$ (but see also Ref. [21] and J. Gegelia's contribution to this conference).

Crucial for an EFT are regulator independence (within the range of validity of the EFT) and a power counting scheme that allows for order-by-order improvement with decreasing truncation error. The purpose of renormalization is to achieve this regulator independence while maintaining a functional power counting scheme.

Thus, in the spirit of Lepage 22], the cutoff independence should be examined for cutoffs below the hard scale and not beyond. Ranges of cutoff independence within the theoretical error are to be identified using 'Lepage plots' 22. Recently, we have started a systematic investigation of this kind. In our work, we quantify the error of the predictions by calculating the $\chi^{2} /$ datum for the reproduction of the neutron-proton $(n p)$ elastic scattering data as a function of the cutoff parameter $\Lambda$ of the regulator function Eq. (6). We have investigated the predictions by chiral $n p$ potentials at order NLO and NNLO applying Weinberg counting for the counter terms ( $N N$ contact terms). 

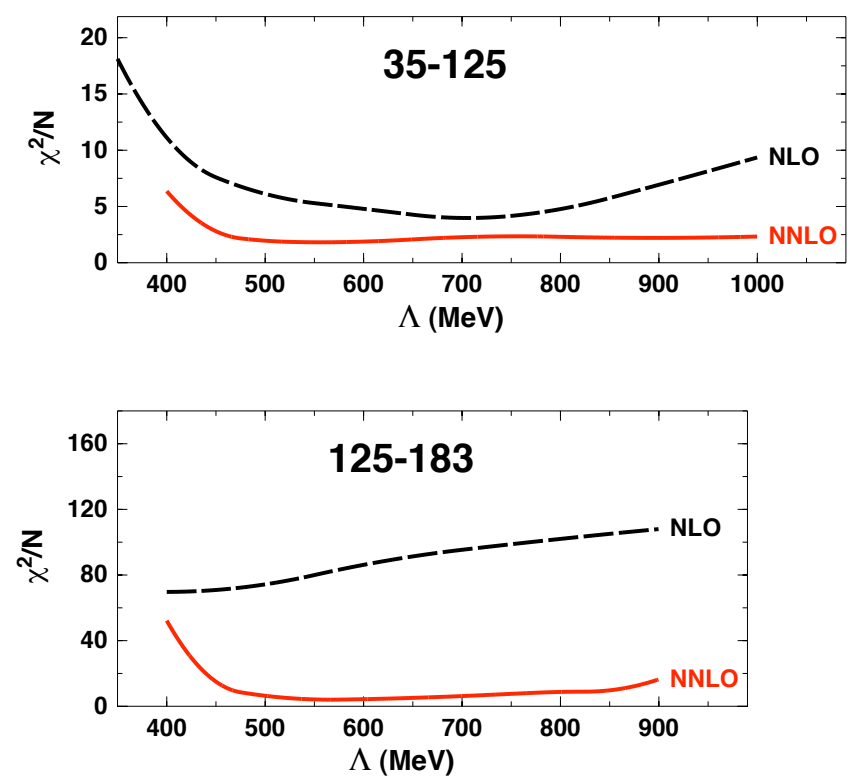

Fig. $1 \chi^{2} /$ datum for the reproduction of the $n p$ data in the energy range $35-125 \mathrm{MeV}$ (upper frame) and $125-183 \mathrm{MeV}$ (lower frame) as a function of the cutoff parameter $\Lambda$ of the regulator function Eq. 66. The (black) dashed curves show the $\chi^{2} /$ datum achieved with $n p$ potentials constructed at order NLO and the (red) solid curves are for NNLO.

We show our results for the energy range $35-125 \mathrm{MeV}$ in the upper frame of Fig. 1 and for $125-183 \mathrm{MeV}$ in the lower frame. It is seen that the reproduction of the $n p$ data at these energies is generally poor at NLO, while at NNLO the $\chi^{2} /$ datum assumes acceptable values (a clear demonstration of order-by-order improvement). Moreover, at NNLO one observes "plateaus" of constant low $\chi^{2}$ for cutoff parameters ranging from 450 to $850 \mathrm{MeV}$. This may be perceived as cutoff independence (and, thus, successful renormalization) in the relevant range of cutoff parameters.

\section{Few-nucleon forces and what is missing}

We will now discuss the other issue we perceive as unfinished and important, namely, subleading chiral few-nucleon forces.

Nuclear three-body forces in ChPT were initially discussed by Weinberg [8]. The three-nucleon force (3NF) at NNLO, was derived by van Kolck [10] and applied, for the first time, in nucleon-deuteron scattering by Epelbaum et al. 23. The leading $4 \mathrm{NF}$ (at $\mathrm{N}^{3} \mathrm{LO}$ ) was constructed by Epelbaum 24 and found to contribute in the order of $0.1 \mathrm{MeV}$ to the ${ }^{4} \mathrm{He}$ binding energy (total ${ }^{4} \mathrm{He}$ binding energy: $28.3 \mathrm{MeV}$ ) in a preliminary calculation [25], confirming the traditional assumption that $4 \mathrm{NF}$ are essentially negligible. Therefore, the focus is on 3NF.

For the order of a $3 \mathrm{NF}$, we have

$$
\nu=2+2 L+\sum_{i} \Delta_{i}
$$


where $L$ denotes the number of loops and $\Delta_{i}$ is the vertex index. We will use this equation to analyze $3 \mathrm{NF}$ contributions order by order. The first non-vanishing $3 \mathrm{NF}$ occurs at $\nu=3(\mathrm{NNLO})$, which is obtained when there are no loops $(L=0)$ and $\sum_{i} \Delta_{i}=1$, i.e., $\Delta_{i}=1$ for one vertex while $\Delta_{i}=0$ for all other vertices. There are three topologies which fulfill this condition, known as the two-pion exchange (2PE), one-pion exchange (1PE), and contact graphs.

The $3 \mathrm{NF}$ at NNLO has been applied in calculations of few-nucleon reactions [26, structure of light- and medium-mass nuclei $[27,28,29,30,31,32$, and nuclear and neutron matter [33, 34,35,36] with a great deal of success. However, the famous ' $A y$ puzzle' of nucleon-deuteron scattering [23] and the analogous problem with the analyzing power in $p-{ }^{3} \mathrm{He}$ scattering [37] is not resolved. Furthermore, the spectra of light nuclei leave room for improvement [27. Since we are dealing with a perturbation theory, it is natural to turn to the next order when looking for improvements.

The next order is $\mathrm{N}^{3} \mathrm{LO}$, where we have loop and tree diagrams. For the loops, we have $L=1$ and, therefore, all $\Delta_{i}$ have to be zero to ensure $\nu=4$. Thus, these one-loop $3 \mathrm{NF}$ diagrams can include only leading order vertices, the parameters of which are fixed from $\pi N$ and $N N$ analysis. One sub-group of these diagrams (the 2PE graphs) has been calculated by Ishikawa and Robilotta [38, and the other topologies have been evaluated by the Bochum-Bonn group 39 40. The $\mathrm{N}^{3} \mathrm{LO} 2 \mathrm{PE} 3 \mathrm{NF}$ has been applied in the calculation of nucleon-deuteron observables in Ref. 38] causing little impact. Very recently, the long-range part of the chiral $\mathrm{N}^{3} \mathrm{LO} 3 \mathrm{NF}$ has been tested in the triton [41] and in three-nucleon scattering [42] yielding only moderate effects. The long- and shortrange parts of this force have been used in neutron matter calculations (together with the $\mathrm{N}^{3} \mathrm{LO} 4 \mathrm{NF}$ ) producing relatively large contributions from the $3 \mathrm{NF}$ [3]. Thus, the ultimate assessment of the $\mathrm{N}^{3} \mathrm{LO} 3 \mathrm{NF}$ is still outstanding and will require more fewand many-body applications.

In the meantime, it is of interest to take already a look at the next order of $3 \mathrm{NFs}$, which is $\mathrm{N}^{4} \mathrm{LO}$ or $\nu=5$ (of the $\Delta$-less theory to which the present discussion is restricted because of lack of space). The loop contributions that occur at this order are obtained by replacing in the $\mathrm{N}^{3} \mathrm{LO}$ loops one vertex by a $\Delta_{i}=1$ vertex (with LEC $c_{i}$ ), which is why these loops may be more sizable than the $\mathrm{N}^{3} \mathrm{LO}$ loops. The $2 \mathrm{PE}$ topology has already been evaluated [44 and turns out to be of modest size; moreover, it can be handled in a practical way by summing it up together with the $2 \mathrm{PE}$ topologies at $\mathrm{NNLO}$ and $\mathrm{N}^{3} \mathrm{LO}$ 44. However, there are four more loop topologies, which are very involved and that have not been worked out yet. Finally, a tree topology at $\mathrm{N}^{4} \mathrm{LO}$ provides a new set of $3 \mathrm{~N}$ contact interactions, which have recently been derived by the Pisa group [45. Contact terms are typically simple (as compared to loop diagrams) and their coefficients are unconstrained (except for naturalness). Therefore, it would be an attractive project to test some terms (in particular, the spin-orbit terms) of the $N^{4} L O$ contact $3 N F$ [45] in calculations of few-body reactions (specifically, the p-d and $p^{3}{ }^{3} \mathrm{He} \mathrm{A}_{y}$ ) and spectra of light nuclei.

\section{Conclusions and Outlook}

The past 15 years have seen great progress in our understanding of nuclear forces in terms of low-energy QCD. Key to this development was the realization that low-energy QCD is equivalent to an effective field theory (EFT) which allows for a perturbative expansion that has become known as chiral perturbation theory (ChPT). In this frame- 
work, two- and many-body forces emerge on an equal footing and the empirical fact that nuclear many-body forces are substantially weaker then the two-nucleon force is explained automatically.

In spite of the great progress and success of the past 15 years, there are still some unresolved issues. One problem is the proper renormalization of the chiral two- and many-nucleon potentials, where systematic investigations are already under way (cf. Sec. 2).

The other unfinished business are the few-nucleon forces beyond NNLO ("subleading few-nucleon forces") which are needed to hopefully resolve some important outstanding nuclear structure problems. At orders $\mathrm{N}^{3} \mathrm{LO}$ and $\mathrm{N}^{4} \mathrm{LO}$ very many new $3 \mathrm{NF}$ structures appear, some of which have already been tested. However, in view of the multitude of $3 \mathrm{NF}$ topologies it will take a while until we will have a proper overview of impact and convergence of these contributions.

If the open issues discussed in this paper will be resolved within the next few years, then, after 80 years of desperate struggle, we may finally claim that the nuclear force problem is essentially under control. The greatest beneficiaries of such progress will be the fields of exact few-nucleon calculations and ab initio nuclear structure physics.

Acknowledgements This work was supported in part by the U.S. Department of Energy under Grant No. DE-FG02-03ER41270. The work of D. R. E. was funded by the Ministerio de Ciencia y Tecnología under Contract No. FPA2007-65748, the Junta de Castilla y León under Contract No. GR12, and the European Community-Research Infrastructure Integrating Activity "Study of Strongly Interacting Matter" (HadronPhysics2 Grant No. 227431).

\section{References}

1. Yukawa, H.: On the interaction of elementary particles. Proc. Phys. Math. Soc. Japan 17, 48 (1935).

2. Prog. Theor. Phys. (Kyoto), Supplement 3 (1956).

3. Taketani, M., Machida, S., Onuma, S.: Prog. Theor. Phys. (Kyoto) 7, 45 (1952).

4. Machleidt, R.: The Meson Theory of Nuclear Forces and Nuclear Structure. Adv. Nucl. Phys. 19, 189 (1989)

5. Machleidt, R., Holinde, K., Elster, Ch.: The Bonn Meson-Exchange Model for The Nucleon-Nucleon Interaction. Phys. Rep. 149, 1 (1987)

6. Weinberg, S.: Phenomenological Lagrangians. Physica 96A, 327 (1979)

7. Weinberg, S.: Effective Chiral Lagrangians for Nucleon-Pion Interactions and Nuclear Forces. Nucl. Phys. B363, 3 (1991)

8. Weinberg, S.: Three-body interactions among nucleons and pions. Phys. Lett. B 295, 114 (1992)

9. Ordóñez, C., Ray, L., van Kolck, U.: Nucleon-Nucleon Potential from an Effective Chiral Lagrangian. Phys. Rev. Lett. 72, 1982 (1994)

10. van Kolck, U.: Few-nucleon forces from chiral Lagrangians. Phys. Rev. C 49, 2932 (1994)

11. Kaiser, N., Brockmann, R., Weise, W.: Peripheral nucleon-nucleon phase shifts and chiral symmetry. Nucl. Phys. A625, 758 (1997)

12. Entem, D.R., Machleidt, R.: Accurate charge-dependent nucleon-nucleon potential at fourth order of chiral perturbation theory. Phys. Rev. C 68, 041001 (2003)

13. Epelbaum, E., Glöckle, W., Meißner, U.-G.: The two-nucleon system at next-to-next-tonext-to-leading order. Nucl. Phys. A747, 362 (2005)

14. Machleidt, R., Entem, D.R.: Chiral effective field theory and nuclear forces. Phys. Rep. 503, 1 (2011), and references therein

15. Nogga, A., Timmermans, R.G.E., van Kolck, U.: Renormalization of one-pion exchange and power counting. Phys. Rev. C 72, 054006 (2005)

16. Yang, C.-J., Elster, Ch., Phillips, D.R.: Subtractive renormalization of the chiral potentials up to next-to-next-to-leading order in higher NN partial waves. Phys. Rev. C 80, 034002 (2009) 
17. Pavon Valderrama, M., Ruiz Arriola, E.: Renormalization of the $N N$ interaction with a chiral two-pion exchange potential. II. Noncentral phases. Phys. Rev. C 74, 064004 (2006)

18. Entem, D.R., Ruiz Arriola, E., Pavón Valderrama, M., Machleidt, R.: Renormalization of chiral two-pion exchange $N N$ interactions: Momentum space versus coordinate space. Phys. Rev. C 77, 044006 (2008)

19. Zeoli, Ch., Machleidt, R., Entem, D.R.: Infinite-cutoff renormalization of the chiral nucleon-nucleon interaction up to N ${ }^{3}$ LO. Few-Body Syst. DOI 10.1007/s00601-012-0481-4, arXiv:1208.2657 [nucl-th]

20. Epelbaum, E., Gegelia, J.: Regularization, renormalization and 'peratization' in effective field theory for two nucleons. Eur. Phys. J. A41, 341 (2009)

21. Epelbaum, E., Gegelia, J.: Weinberg's approach to nucleon-nucleon scattering revisited. Phys. Lett B 716, 338 (2012)

22. Lepage, G.P.: How to Renormalize the Schrödinger Equation. arXiv:nucl-th/9706029

23. Epelbaum, E. et al.: Three-nucleon forces from chiral effective field theory. Phys. Rev. C 66, $064001(2002)$

24. Epelbaum, E.: Four-nucleon force using the method of unitary transformation. Eur. Phys. J. A34, 197 (2007)

25. Rozpedzik, D. et al.: A first estimation of chiral four-nucleon force effects in ${ }^{4}$ He. Acta Phys. Polon. B37, 2889 (2006)

26. Kalantar-Nayestanaki, N., Epelbaum, E., Messchendorp, J.G., Nogga, A.: Signatures of three-nucleon interactions in few-nucleon systems. Rep. Prog. Phys. 75, 016301 (2012)

27. Navratil, P., Gueorguiev, V.G., Vary, J.P., Ormand, W.E., Nogga, A.: Structure of $A=$ $10-13$ nuclei with two- plus three-nucleon interactions from chiral effective field theory. Phys. Rev. Lett. 99, 042501 (2007)

28. Otsuka, T., Susuki, T., Holt, J.D., Schwenk, A., Akaishi, Y.:, Three-body forces and the limit on oxygen isotopes. Phys. Rev. Lett. 105, 032501 (2010)

29. Holt, J.W., Kaiser, N., Weise, W., Chiral three-nucleon interaction and the ${ }^{14}$ C-dating $\beta$ decay. Phys. Rev. C 79, 054331 (2009).

30. Roth, R., Binder, S., Vobig, K., Calci, A., Langhammer, J., Navratil, P.,: Medium-mass nuclei with normal-ordered chiral NN+3N interactions. Phys. Rev. Lett. 109, 052501 (2012)

31. Hagen, G., Hjorth-Jensen, M., Jansen, G.R., Machleidt, R., Papenbrock, T.: Continuum effects and three-nucleon forces in neutron-rich oxygen isotopes. Phys. Rev. Lett. 108, 242501 (2012).

32. Hagen, G., Hjorth-Jensen, M., Jansen, G.R., Machleidt, R., Papenbrock, T.: Evolution of shell structure in neutron-rich calcium isotopes. Phys. Rev. Lett. 109, 032502 (2012).

33. Hebeler K., Schwenk, A.: Chiral three-nucleon forces and neutron matter. Phys. Rev. C 82, $014314(2010)$

34. Hebeler, K., Bogner, S.K., Furnstahl, R.J., Nogga, A., Schwenk, A: Improved nuclear matter from chiral low-momentum interactions, Phys. Rev. C 83, 031301(R) (2011)

35. Sammarruca, F., Chen, B., Coraggio, L., Itaco, N., Machleidt, R.: Dirac-BruecknerHartree-Fock versus chiral effective field theory. arXiv:1209.5001 [nucl-th]

36. Coraggio, L., Holt, J.W., Itaco, N., Machleidt, R., Sammarruca, F.: Regulator independence of neutron-matter predictions with chiral interactions. arXiv:1209.5537 [nucl-th]

37. Viviani, M., Giarlanda, L., Kievsky, A., Marcucci, L.E., Rosati, S.: Proton- ${ }^{3}$ He elastic scattering at low energies and the " $A_{y}$ Puzzle". arXiv:1004.1306 [nucl-th]

38. Ishikawa, S., Robilotta, M.R.: Two-pion exchange three-nucleon potential: $\mathcal{O}\left(q^{4}\right)$ chiral expansion. Phys. Rev. C 76, 014006 (2007)

39. Bernard, V., Epelbaum, E., Krebs, H., Meißner, U.-G.: Subleading contributions to the chiral three-nucleon force I: long-range terms. Phys. Rev. C 77, 064004 (2008)

40. Bernard, V., Epelbaum, E., Krebs, H., Meißner, U.-G.: Subleading contributions to the chiral three-nucleon force II: short-range terms and relativistic corrections. Phys. Rev. C 84, 054001 (2011)

41. Skibinski, R., et al.: The triton with long-range chiral $\mathrm{N}^{3} \mathrm{LO}$ three nucleon forces. Phys. Rev. C 84, 054005 (2011)

42. Witala, H.: Calculations of three-nucleon reactions. Proceedings of this conference

43. Tews, I., Krüger, T., Hebeler, K., Schwenk, A.: Neutron matter at next-to-next-to-nextto-leading order in chiral effective field theory, arXiv:1206.0025 [nucl-th]

44. Krebs, H., Gasparyan, A., Epelbaum, E.: Chiral three-nucleon force at $\mathrm{N}^{4} \mathrm{LO}$ I: Longestrange contributions. Phys. Rev. C 85, 054006 (2012)

45. Girlanda, L., Kievsky, A., Viviani, M.: Subleading contributions to the three-nucleon contact interaction. Phys. Rev. C 84, 014001 (2011) 